\title{
ANÁLISE DA DOMINAÇÃO DE MEMBROS TOMADORES OU POUPADORES DE RECURSOS NAS COOPERATIVAS DE CRÉDITO MINEIRAS
}

\author{
Valéria Gama Fully Bressan * \\ Marcelo José Braga ${ }^{\dagger}$ \\ Aureliano Angel Bressan ${ }^{\ddagger}$
}

\begin{abstract}
Resumo
O objetivo deste estudo foi identificar o comportamento de dominação no sistema Sicoob-Crediminas do estado de Minas Gerais. Cooperativas dominadas por tomadores são aquelas que oferecem baixas taxas de juros para empréstimos e baixas taxas para poupança, ao passo que cooperativas dominadas por poupadores praticam taxas mais elevadas, tanto para poupança quanto para empréstimo. Nesta perspectiva, os resultados do estudo indicam que o sistema Sicoob-Crediminas é dominado por membros tomadores, apresentando melhores taxas de empréstimo e poupança se comparadas às taxas praticadas pelo sistema bancário.
\end{abstract}

Palavras-chave: Cooperativas de Crédito; Análise de Dominação; Análise Econômica de cooperativas.

\begin{abstract}
The aim of this study was to identify the dominance behavior in credit unions affiliated to the Sicoob-Crediminas system in Minas Gerais state, Brazil. Borrower-dominated unions are those that offer low borrowing rates and also low saving rates, while saver-dominated unions practice higher rates for savings as well as for loans. The results indicate that Sicoob-Crediminas unions are dominated by borrowing members, with better savings and loan rates, in comparison to the rates committed by the banking system.
\end{abstract}

Keywords: Credit Unions; Dominance Analysis; Economic Analysis of Credit Unions.

JEL classification: P13, G21

\footnotetext{
* Universidade Federal de Minas Gerais. E-mail: valeria.fully@gmail.com

† Universidade Federal de Viçosa. E-mail: mjbraga@ufv.br

‡ Universidade Federal de Minas Gerais. E-mail: bressan@face.ufmg.br
} 


\section{Introdução}

Nas cooperativas de crédito os cooperados são, ao mesmo tempo, ofertantes e usuários dos fundos de empréstimos. Assim, de acordo com Taylor (1971), a análise econômica das cooperativas deve considerar importantes relações, tais como: a existência de um vínculo econômico entre a cooperativa e seus membros e, paralelamente, a ligação entre a cooperativa e o mercado.

Em função destas particularidades, Taylor (1971) investigou a importância social e as implicações econômicas deste tipo de organização. $\mathrm{O}$ autor comenta que uma das características das cooperativas não é o lucro individual, mas sim o alcance dos objetivos econômicos e sociais do grupo. O comportamento econômico nas cooperativas pode então ser visto como uma extensão do comportamento econômico dos seus membros, oposto ao comportamento individual independente.

A cooperativa de crédito é então uma forma de organização socioeconômica, a qual é uma fonte de recursos não somente para consumidores de crédito, mas também para aplicadores. Alguns cooperados podem usar estas cooperativas exclusivamente para aplicações de recursos financeiros, enquanto outros as consideram como fonte de consumo de crédito. $\mathrm{O}$ fato é que os grupos dentro das cooperativas de crédito possuem diferentes relações econômicas, e estas implicam em certo grau de conflito de interesses entre os membros.

De modo geral, os membros aplicadores querem receber as mais altas remunerações possíveis sobre seus depósitos; ao passo que membros tomadores querem minimizar as taxas de juros sobre os empréstimos. A cooperativa de crédito não pode simultaneamente maximizar os retornos pagos para os membros aplicadores e minimizar as taxas sobre os empréstimos para os membros tomadores. Assim, pode-se desenvolver um conflito entre os membros aplicadores e os membros tomadores (Patin \& McNiel 1991b). Por este motivo, os modelos aplicados a sociedades de capital baseados na maximização de lucro não podem ser aplicados diretamente às cooperativas de crédito (Smith et al. 1981).

Dada a relevância deste tema, Black \& Dugger (1981) destacaram que algumas cooperativas de crédito são então classificadas como dominadas por tomadores enquanto outras seriam dominadas por aplicadores, e que este perfil tem implicações nas formas de gerenciamento e obtenção de ganhos por parte das cooperativas. Por este motivo, esta temática é um aspecto importante para a avaliação de cooperativas de crédito, o que foi abordado por vários autores, tais como Walker \& Chandler (1977), Taylor (1979), Smith et al. (1981), Black \& Dugger (1981), Smith (1986), Patin \& McNiel (1991a,b), Emmons \& Mueller (1997), Leggett \& Stewart (1999) e Goddard \& Wilson (2005)

Neste contexto, não foram constatados na literatura nacional estudos que avaliassem a existência de algum tipo de dominação nas cooperativas de crédito brasileiras, seja por membros aplicadores ou por membros tomadores de recursos, ou mesmo se existe uma neutralidade em termos de comportamento dos cooperados.

O objetivo do presente estudo é então identificar o comportamento de dominação de membros tomadores ou de membros aplicadores de recursos nas cooperativas de crédito filiadas ao Sicoob-Crediminas e, em havendo dominação, avaliar quais os determinantes desta dominação. Com isso, pretende-se então contribuir para a discussão desta temática em relação às cooperativas de crédito mineiras, especificamente aquelas filiadas ao Sicoob-Crediminas, que 
constituem parte integrante do Sistema de Cooperativas de Crédito do Brasil $(\text { Sicoob })^{1}$.

A escolha do Sicoob está fundamentada em sua representatividade e abrangência nacional, atuando em 21 estados brasileiros e no Distrito Federal, disponibilizando produtos e serviços financeiros,o que é composto por 14 cooperativas centrais, 633 cooperativas singulares de crédito, 1.144 postos de atendimento cooperativo (PACs) e que conta ainda com a participação de mais de 1,7 milhão de associados e pela Confederação Nacional de Cooperativas de Crédito (Sicoob-Brasil).

O estudo atual avaliou as cooperativas de crédito filiadas ao Sicoob-Crediminas no período de 1995 a 2008, totalizando 117 cooperativas. Ressalta-se que em 2008 havia apenas 97 cooperativas filiadas. Essa redução foi decorrente de fusões, incorporações, entre outros fatores, e essas 97 cooperativas representam aproximadamente $50 \%$ das cooperativas de crédito mineiras filiadas ao Sicoob. Assim, a amostra do presente estudo foi formada por 16.010 observações mensais, referente ao período de janeiro de 1995 a maio de 2008 .

O artigo está organizado em quatro seções, além desta introdução. A próxima seção apresenta a revisão de literatura. A seguir, apresenta-se a metodologia empregada na pesquisa. A quarta seção discute os resultados encontrados. A última seção apresenta as principais implicações do estudo, bem como recomendações para pesquisas futuras.

\section{Revisão da Literatura}

\subsection{Cooperados: Tomadores-líquidos ou Aplicadores-líquidos?}

Nos estudos de Teoria da Firma, é aceitável assumir unanimidade entre os acionistas com respeito à função objetivo, na qual tipicamente busca-se maximizar o valor da firma, o retorno sobre os ativos, ou simplesmente a maximização de lucros. As cooperativas de crédito, entretanto, são um exemplo de organização na qual a pressuposição "unânime" não é necessariamente aplicável (Smith 1986). Isto porque, nestas organizações os membros são: i) proprietários da organização (equity holders), ii) consumidores do seu produto (crédito), iii) ofertantes do seu insumo primário (fundos que são emprestados) e iv) em muitos casos, os administradores destas operações (Patin \& McNiel 1991a). Nesse sentido, a cooperativa de crédito pode ser modelada como maximizadora dos interesses dos seus membros quando opera com níveis de produto que minimizam o hiato entre a taxa cobrada sobre os empréstimos e a taxa paga sobre as poupanças.

Segundo Leggett \& Stewart (1999), considera-se que as cooperativas de crédito que oferecem baixas taxas sobre os empréstimos e baixas taxas de remuneração sobre os depósitos são orientadas para os tomadores, já aquelas que praticam altas taxas de remuneração sobre os depósitos e consequentemente altas taxas sobre os empréstimos são orientadas para os aplicadores.

\footnotetext{
${ }^{1}$ No Brasil, as cooperativas são consideradas intermediários financeiros. Para obter ganhos de escala no processo administrativo e na supervisão financeira, as cooperativas de crédito se organizam em singulares, centrais e confederações, na forma de rede. A central é formada por um conjunto de singulares a ela vinculadas. Já, a confederação é constituída por um conjunto de centrais vinculadas. De acordo com dados de Soares \& Melo Sobrinho (2007), o Sicoob destacase o como o maior sistema dentre os demais existentes no Brasil - Sicredi, Unicred, Ancosol, Cooperativas Independentes e outros sistemas - por abranger $46,32 \%$ das cooperativas singulares brasileiras.
} 
As cooperativas de crédito que estabelecem um equilíbrio entre as taxas sobre os empréstimos e sobre os depósitos são designadas como cooperativas de crédito neutras.

De acordo com Patin \& McNiel (1991b), os membros aplicadores querem receber as mais altas remunerações possíveis sobre seus depósitos na cooperativa de crédito. Por outro lado, os membros tomadores querem minimizar as taxas de juros sobre os empréstimos. Todavia, a cooperativa de crédito não pode simultaneamente maximizar os retornos pagos para os membros aplicadores e minimizar as taxas sobre os empréstimos para os membros tomadores. Assim, pode-se desenvolver um conflito entre os aplicadores-líquidos e os tomadores-líquidos dentro de uma mesma unidade cooperativa.

Esta percepção é corroborada por Smith (1986), ao afirmar que desde que a totalidade dos membros possa ser dividida em tomadores-líquidos e aplicadores-líquidos existe um conflito inerente. Os tomadores naturalmente irão preferir objetivos estabelecidos em termos de baixas taxas de juros para a obtenção de empréstimos, enquanto os aplicadores irão preferir altas taxas pagas sobre os depósitos. Por este motivo, os modelos aplicados a firmas financeiras, baseados na maximização do lucro, não podem ser aplicados diretamente para as cooperativas de crédito (Smith et al. 1981).

Este conflito entre membros tomadores e membros aplicadores foi apresentado também por Walker \& Chandler (1977), ao afirmarem que para uma cooperativa de crédito manter baixas taxas sobre os empréstimos, seria necessário que a mesma limitasse a capacidade de distribuir sobras; ao passo que se a cooperativa mantivesse altas sobras poderia ser necessário praticar altas taxas sobre os empréstimos. Dessa forma, a resolução deste conflito - a concorrência de taxas entre estes dois grupos na cooperativa - não é uma tarefa simples em um ambiente não restrito, e se torna mais difícil devido às restrições regulatórias às quais a administração das cooperativas de crédito deve satisfazer. De acordo com estes autores, dois aspectos regulatórios - teto máximo para taxa sobre empréstimos e teto máximo para distribuição de sobras - impedem a cooperativa de tratar imparcialmente a alocação dos benefícios monetários líquidos para os membros, resultando em um viés pró-tomadores.

Smith (1986) investigou se as cooperativas de crédito americanas tendem a ser dominadas por tomadores-líquidos ou por aplicadores-líquidos. Os resultados obtidos indicaram que, na média, as cooperativas norte-americanas tendem a um comportamento neutro. Já Fischer \& Desrochers (1999) apud Fischer (2000) encontraram evidências na Colômbia de que as cooperativas de crédito podem ser dominadas por tomadores ou aplicadores e que essa diferença reflete no risco do portfólio de crédito.

Conforme Black \& Dugger (1981), nas cooperativas de crédito, os membros aplicadores são também tomadores. No entanto, em algumas cooperativas pode haver diferentes perfis de aplicadores e tomadores. Assim, algumas cooperativas são referendadas como dominadas por tomadores, enquanto outras como dominadas por aplicadores. Além disso, a maneira como a cooperativa obtém seus ganhos tem implicações no perfil da cooperativa de crédito. Pode haver restituição de juros indiretamente, através da distribuição de sobras em cooperativas de crédito dominadas por membros tomadores, e aumento de sobras incorporadas ao capital em instituições dominadas por aplicadores. Entretanto, sobras crescentes não estariam disponíveis se as cooperativas de crédito já estivessem distribuindo o máximo admissível de sobras.

Taylor (1979) faz uma analogia das cooperativas de crédito considerando 
o perfil de seus membros, em relação a outras formas de organização cooperativista. Assim, cooperados que são exclusivamente tomadores de crédito comportam-se como membros de cooperativas de consumo, e aqueles que são aplicadores são similares aos cooperados das cooperativas agropecuárias. Se a diretoria eleita dá o mesmo grau de importância aos objetivos de ambos os grupos, a cooperativa buscará pagar as mais altas taxas sobre os recursos poupados e cobrará baixas taxas sobre os empréstimos, considerando a natureza da demanda por empréstimos, da oferta de poupança e dos custos operacionais. É importante assumir que os cooperados são livres para a exercer demanda de empréstimos e a oferta de poupanças, devido às taxas praticadas pelas cooperativas.

De acordo com Patin \& McNiel (1991a), os modelos teóricos para o comportamento das cooperativas de crédito sugerem que estas podem ser dominadas por tomadores, aplicadores e/ou serem neutras. No estudo realizado em 1991, os autores mediram os benefícios monetários dos grupos de membros nas cooperativas de crédito americanas no período de 1984/85, constatando que as cooperativas de crédito distribuíram maiores benefícios monetários para os aplicadores do que para os tomadores. Entretanto, os autores questionaram se a magnitude do viés em prol dos aplicadores é grande o suficiente para afetar a tomada de decisão dos policy-makers. A justificativa econômica para o movimento de cooperativas de crédito é realçada pelo fato de que a neutralidade foi o tipo de comportamento mais verificado no estudo. Patin \& McNiel (1991b) analisando as cooperativas de crédito americanas em 1985, constataram que a maioria exibe regularmente comportamento neutro, apesar da evidência da presença de cooperativas de crédito com comportamento direcionado tanto para aplicadores quanto para tomadores.

Leggett \& Stewart (1999) analisaram os efeitos da alocação de benefícios monetários líquidos, tanto para aplicadores quanto para tomadores de recursos, considerando as características ${ }^{2}$ das cooperativas "fechadas" e das cooperativas "abertas" no ano de 1997, nas 25 maiores regiões metropolitanas dos EUA. De acordo com estes autores, a partir de 1982 foi permitido, nos EUA, a inclusão de cooperados de grupos não relacionados, ou seja, cooperativas abertas. No caso do Brasil, as cooperativas abertas foram autorizadas pelo Banco Central do Brasil em 2003. A adição de novos membros de grupos que não são relacionados com o "core-group" aumenta o interesse de questões relacionadas à governança/controle e à distribuição dos benefícios entre os membros. Isso porque, segundo a National Association of Federal Credit Unions (NAFCU), os membros não vinculadores ao "core-group" são provavelmente mais propensos a serem tomadores de crédito que os membros do "core-group". Os autores concluíram que, em média, as cooperativas de crédito são orientadas para os aplicadores e que as cooperativas fechadas têm uma maior orientação para aplicadores em relação às abertas.

Kohers \& Mullis (1990) apud Goddard \& Wilson (2005) sugerem que as co-

\footnotetext{
${ }^{2}$ Cooperativas de crédito fechadas indicam aquelas em que os membros possuem características similares, ou seja, pertencem a uma mesma categoria profissional, ou estão vinculadas a uma mesma instituição. Estas são cooperativas de crédito onde não se tem a livre admissão. Cooperativas de crédito abertas são aquelas que permitem a livre admissão dos associados, ou seja não é necessário nenhum vínculo entre os cooperados. No Brasil, as cooperativas de crédito de livre admissão foram permitidas em 2003, de acordo com a BANCO CENTRAL DO BRASIL. Resolução 3.106/2003. (2007). No entanto, existem restrições para esta livre admissão Para maiores detalhes veja BANCO CENTRAL DO BRASIL. Resolução 3.106/2003. (2007).
} 
operativas de crédito mais antigas tendem a pagar taxas mais altas e são orientadas para os aplicadores, enquanto as mais jovens praticam taxas mais baixas e são orientadas para os tomadores porque estão sujeitas à restrição de sustentabilidade de sobras não negativas. Goddard \& Wilson (2005), em seu estudo sobre tamanho, idade e crescimento das cooperativas de crédito americanas, constataram evidências consistentes com aquelas encontradas por Kohers \& Mullis (1990), isto é, de que cooperativas mais jovens são orientadas para tomadores e as mais antigas para os aplicadores. As cooperativas de crédito orientadas para os tomadores tendem a minimizar as taxas sobre os empréstimos, resultando em forte demanda de empréstimo, crescimento do ativo e aumento no número de associados.

De acordo com Bonus \& Schmidt (1990) apud Emmons \& Mueller (1997), os bancos cooperativos na Alemanha foram fundados em meados do século XIX por membros cujo interesse primário era a obtenção de crédito a baixo custo. Ou seja, estas cooperativas eram dominadas por tomadores e os associados avaliavam muito mais a disponibilidade de crédito do que a possibilidade de ganhos com sobras (Emmons \& Mueller 1997).

No entanto, para estes autores, as características dos membros das cooperativas mudaram, bem como as políticas dos bancos cooperativos. Quando o membro-mediano da cooperativa tornou-se mais interessado em ganhos sobre seu investimento do que na possibilidade de tomar emprestado a taxas inferiores às praticadas no mercado, a estrutura democrática de voto permitiu uma mudança na política da cooperativa. Por meio de um aumento no preço do crédito em direção a um nível eficiente, a cooperativa foi capaz de aumentar sua receita e distribuir as sobras de forma competitiva. Isso permitiu aos bancos cooperativos manterem seus membros aplicadores apesar de o núcleo de membros iniciais - os tomadores - estar menos bem servidos que antes. Todavia, a ameaça de saída dos membros tomadores serve como um forte mecanismo disciplinador, fazendo com que o preço do crédito não seja maior que o nível praticado no mercado (Emmons \& Mueller 1997).

\section{Metodologia}

\subsection{Avaliação da existência de dominação nas cooperativas de crédito.}

Tomando por base os fundamentos da teoria da cooperação, advinda do trabalho de Taylor (1971) e posteriormente ampliada por Spencer (1996), e os trabalhos de Walker \& Chandler (1977), Smith et al. (1981), Smith $(1984,1986)$, Patin \& McNiel $(1991 b, a)$ e Leggett \& Stewart (1999), que reconhecem que o conflito entre os membros pode afetar a maneira como as cooperativas de crédito operam, três possibilidades de resultados são geralmente identificadas e modeladas na literatura: cooperativas dominadas por tomadores, aplicadores ou neutras.

Os trabalhos teóricos de Walker \& Chandler (1977), Smith et al. (1981) e Smith (1984) sugerem que o comportamento dominante refletirá mais diretamente no sentido da cooperativa de crédito alocar os resultados entre seus membros, tomadores-líquidos e aplicadores-líquidos, e estes resultados podem ser medidos em termos monetários.

De acordo com Walker \& Chandler (1977), os benefícios das cooperativas de crédito podem ser divididos em monetários e não monetários. Os benefí- 
cios não monetários, tais como: aconselhamento financeiro, comodidade de reembolso de poupança e empréstimos através da folha de pagamento, uso em comum de seguro, seguro de vida, compensação de cheque, entre outros, parecem ser uniformemente distribuídos entre aplicadores e tomadores. Assim, esses autores assumem que os benefícios não monetários são distribuídos equitativamente entre as duas classes de membros e, por isso, dedicam maior atenção para a alocação de benefícios monetários. Esta mesma hipótese é assumida por outros autores (Smith et al. 1981, Smith 1984, Patin \& McNiel 1991a, Leggett \& Stewart 1999) e também será incorporada para o presente trabalho. No caso brasileiro, o pressuposto é plausível de ser adotado, visto que as cooperativas são constituídas a partir dos valores de igualdade, democracia e solidariedade. Desse modo, na prática dos seus valores e princípios, os benefícios não monetários são alocados equitativamente entre os membros.

Os benefícios monetários líquidos recebidos pelos aplicadores nas cooperativas de crédito podem ser medidos pelo produto entre o nível de aplicação e a diferença entre a taxa de remuneração da cooperativa e a melhor alternativa de mercado disponível para tipos similares de aplicação. Esta medida leva em conta o custo de oportunidade para os membros quando poupam em suas cooperativas de crédito (Walker \& Chandler 1977, Smith et al. 1981, Smith 1984, Patin \& McNiel 1991a). Desde que a maioria das cooperativas de crédito oferte vários tipos de instrumentos de poupança, cada taxa de remuneração das cooperativas de crédito e a melhor alternativa de taxa no mercado podem ser calculadas através de médias ponderadas, conforme Patin e McNiel (1991a). No entanto, devido a algumas restrições de dados para o presente estudo, adaptou-se as equações 1 e 2, apresentadas no estudo de Patin \& McNiel (1991a), que seguem descritas a seguir:

$$
\mathrm{NMBS}=(\text { WADR }- \text { WAMDR }) \mathrm{TS}
$$

Em que:

NMBS $=$ Benefícios monetários líquidos recebidos pelos aplicadores.

WADR = média das taxas pagas pela cooperativa de crédito sobre todos os instrumentos de aplicação oferecidos. Diante da impossibilidade de coletar diretamente nas cooperativas os valores referentes às taxas pagas pelas cooperativas sobre o montante de recursos, utilizou-se como proxy para esta variável a razão entre as despesas pagas pela cooperativa com as aplicações dos cooperados em reais e o total aplicado pelos cooperados em reais.

WAMDR = média das melhores alternativas de taxas pagas pelo mercado sobre os tipos similares de instrumentos de aplicação. Nesta variável, utilizouse a seguinte proxy: Poupança ${ }^{3}$ - rendimento nominal - (\% a.m.). Justificase esta escolha pelo motivo de estas informações, detalhadas por instituições financeiras estarem disponíveis ao público em geral.

$\mathrm{TS}=$ volume total das aplicações em reais.

Os benefícios monetários líquidos advindos dos membros tomadores podem ser medidos pela diferença entre a taxa de empréstimo das cooperativas de crédito e a melhor alternativa de taxa de empréstimo no mercado, multiplicado pelo nível de atividade de empréstimo (Walker \& Chandler (1977), Smith

\footnotetext{
${ }^{3}$ Além da taxa de poupança, os autores testaram a taxa de depósitos a prazo (CDB/RDB-préfixados) - Taxa para pessoas físicas (\%) - \% a.m.; e a taxa de depósitos a prazo (CDB/RDB-pósfixados) - Taxa para pessoas físicas - \% a.m.; e todas geraram o mesmo resultado do indicador. $\mathrm{O}$ leitor interessado pode solicitar aos autores por e-mail os resultados obtidos.
} 
et al. (1981), Smith (1984), Patin \& McNiel (1991a)). A taxa sobre empréstimo da cooperativa deve também ser ajustada de acordo com os gastos com restituição de juros para os tomadores. Desde que a maioria das cooperativas ofereça vários tipos de instrumentos de empréstimos, cada taxa de empréstimo e a melhor taxa alternativa de mercado devem ser calculadas como uma média, assim descrita:

$$
\mathrm{NMBB}=(\text { WAMLR }-\mathrm{WALR}(1-\mathrm{RRT})) \mathrm{TL}
$$

Em que:

NMBB $=$ benefícios monetários líquidos recebidos pelos tomadores.

WAMLR = média das taxas de empréstimos de mercado cobrada por outras instituições sobre instrumentos de dívida similares àqueles oferecidos pelas cooperativas. Nessa variável, utilizou-se a seguinte proxy: Taxa média mensal (pré-fixada) das operações de crédito com recursos livres referenciais para taxa de juros - Crédito pessoal - \% a.m., que são informações disponíveis à população.

WALR = média das taxas de empréstimos cobrada pela cooperativa para todos os tipos de empréstimos aos seus membros. Novamente, como no caso de WADR, utilizou-se a seguinte proxy: [rendas obtidas pela cooperativa (ou taxa cobrada pela cooperativa) sobre os empréstimos em reais / total de empréstimos concedidos em reais].

RRT $=$ proporção da renda de juros sobre empréstimos que é restituída aos tomadores da cooperativa. Nesta variável utilizou-se a seguinte proxy: [renda restituída em reais / rendas obtidas pela cooperativa (ou taxa cobrada pela cooperativa) sobre os empréstimos].

$\mathrm{TL}=$ volume total de empréstimos concedidos aos membros da cooperativa, em reais.

A equação 2 mede o benefício monetário líquido do custo de oportunidade, para os membros tomadores, da decisão de obter recursos da cooperativa, ao invés de obtê-los na melhor fonte alternativa de recursos.

A fim de comparar os benefícios alocados para cada grupo de membros é preciso calcular para cada cooperativa de crédito a diferença (dif) entre NMBS e NMBB, descrita conforme o estudo de Patin \& McNiel (1991a) :

$$
\text { dif }=\text { NMBS }- \text { NMBB }
$$

Uma cooperativa de crédito específica aloca mais benefícios monetários para seus membros aplicadores (tomadores) se o "dif" da cooperativa de crédito for maior (menor) que zero. O valor de dif $=0$ indica que a cooperativa de crédito aloca equitativamente os benefícios entre ambos os grupos de membros.

Sintetizando o "dif" para todas as cooperativas de crédito como o somatório de "dif", $\sum$ dif, é possível determinar se estas alocam o maior nível agregado de benefício monetário para os membros aplicadores $\left(\sum\right.$ dif $\left.>0\right)$ ou para membros tomadores $\left(\sum\right.$ dif $\left.<0\right)$ Se $\sum$ dif $=0$, este resultado não permite inferir que todas as cooperativas são neutras, ou se estas estão divididas entre aquelas dominadas por tomadores e por aplicadores (Patin \& McNiel 1991a).

Patin \& McNiel (1991a, p.774) sugerem um ajustamento para o viés de tamanho a partir do cálculo do grau no qual a cooperativa de crédito aloca benefícios entre os membros no período $t$ por meio do índice de Dominação $\left(\left(I D_{i t}\right)\right.$ calculado pela equação 4$)$. 


$$
I D_{i t}=\frac{d s_{i t}}{s t d_{t}}
$$

Em que

$$
\begin{gathered}
d s_{i t}=\frac{\mathrm{NMBS}_{i t}}{\mathrm{TS}_{i t}}-\frac{\mathrm{NMBB}_{i t}}{\mathrm{TL}_{i t}} \\
s t d_{t}=\sqrt{\frac{\sum_{i=1}^{n} d s_{i t}^{2}}{n-1}}
\end{gathered}
$$

Para a i-ésima cooperativa de crédito, $d s_{i t}$ representa a diferença entre o $\mathrm{NMBS}_{i t}$ por Real poupado e $\mathrm{NMBB}_{i t}$ por Real emprestado, e $s t d_{t}$ é o desviopadrão de $d s_{i t}$ em torno de zero no tempo $t$. Assim, o valor absoluto de $I D_{i t}$ fornece a magnitude do desvio para a i-ésima cooperativa de crédito - em relação ao comportamento neutro $\left(I D_{i t}=0\right)$ no período $t$, com seu sinal indicando ainda se a cooperativa é dominada por poupadores $\left(I D_{i t}>0\right)$ ou tomadores de recursos $\left(I D_{i t}<0\right)$.

De acordo com Smith (1986), um problema para o teste empírico é que não se sabe a priori se os tipos de cooperativas de crédito, com predominância de tomadores ou aplicadores, de fato existem. Esta mesma constatação se aplica às cooperativas de crédito mineiras, uma vez que não foram encontrados trabalhos que fizessem este tipo de análise.

Smith (1986) alerta que se a hipótese nula, de que as cooperativas de crédito são homogêneas com respeito aos seus objetivos, é verdadeira, não deve ser possível a partição de toda a amostra de modo que diferenças significativas e sistemáticas sejam encontradas.

\subsection{Determinantes da dominação nas cooperativas de crédito.}

A partir da obtenção do índice de dominação (ID), serão verificadas quais variáveis explicam o comportamento de dominação nas cooperativas de crédito mineiras filiadas ao Sicoob-Crediminas, assumindo o ID como variável dependente, no modelo de regressão com dados em painel. Na Tabela 1 encontramse as características utilizadas por vários autores para avaliar os determinantes do comportamento de dominação em cooperativas de crédito.

Nota-se, a partir da Tabela 1, que as variáveis "percentual das taxas praticadas", "nível médio de empréstimo e poupança por membro", "distribuição da renda líquida" e "custo médio das operações" estão relacionadas com a composição da variável dependente ID (índice de dominação). As demais variáveis (Tamanho, Empréstimos/Capital Social, Idade, Tipo, PL/Ativo Total, Despesa operacional/Ativo Total, Taxa de Inadimplência e Reserva de Capital) foram incorporadas no presente estudo, como variáveis independentes. A variável "Reservas/Receita Bruta Total reserva de capital" não foi incorporada devido à similaridade das contas contábeis que compõem a variável "Reserva de $\mathrm{Ca}$ pital". A variável "Localização Geográfica" foi excluída da análise porque, de acordo com Gujarati (2006), o modelo de efeitos fixos pode não conseguir identificar o impacto dessa variável que não muda ao longo do tempo ${ }^{4}$.

\footnotetext{
${ }^{4}$ No presente estudo, foi testada a inclusão da variável localização geográfica no modelo com efeitos fixos para avaliar esta proposição apresentada por Gujarati (2006), e a mesma foi confirmada. Por este motivo as variáveis de localização geográfica foram excluídas do processo de estimação.
} 


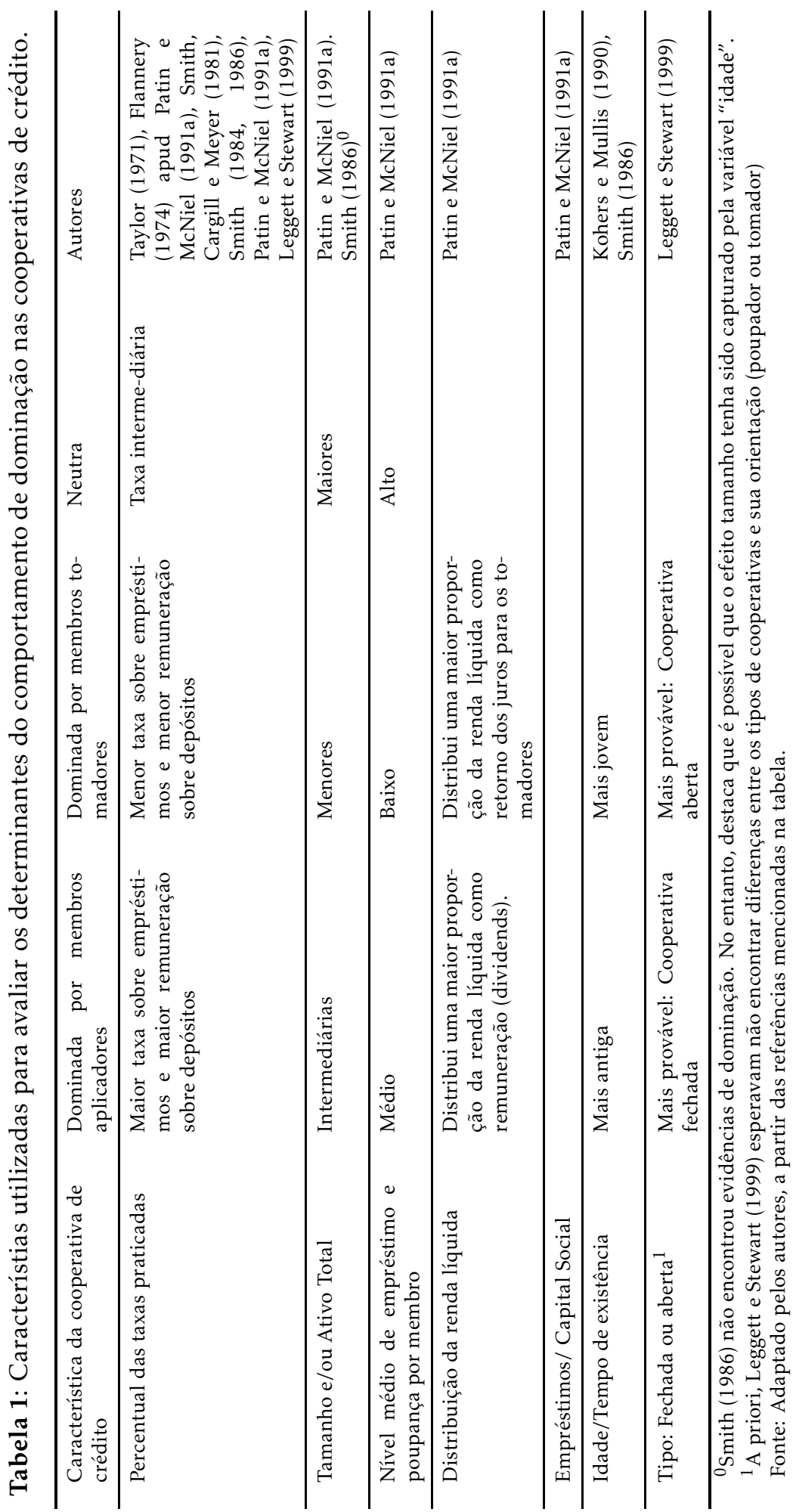




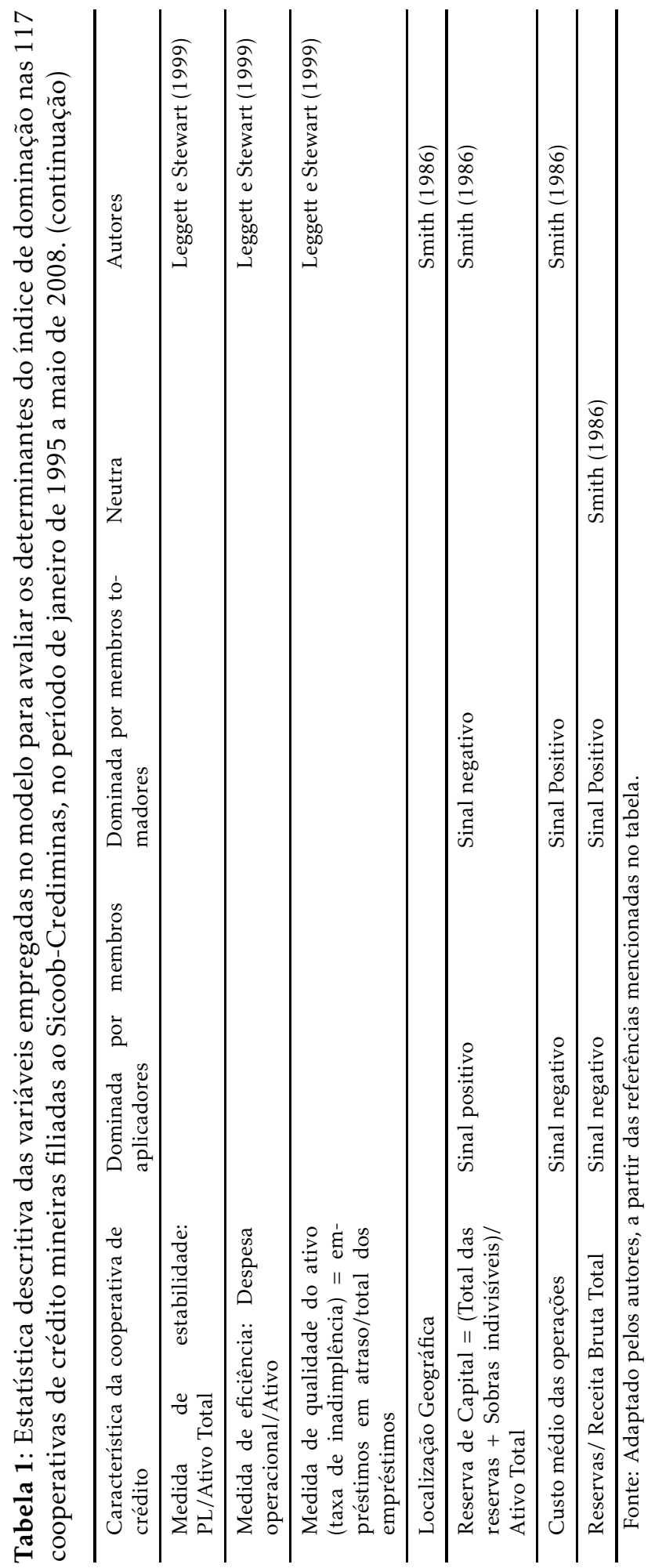




\subsection{Modelo Econométrico: Regressão com Dados em Painel Desbalanceado.}

Os dados em painel têm uma dimensão espacial e outra temporal. Quando cada cooperativa dispõe do mesmo número de dados temporais, o painel caracteriza-se como balanceado (ou equilibrado). Se o número de dados temporais não é o mesmo para todas as cooperativas de crédito, o painel torna-se não balanceado. No presente estudo, utilizam-se relatórios e dados contábeis das cooperativas de crédito filiadas Sicoob-Crediminas que, em vários casos, não possuem informações temporais para todo o período do estudo, o que levou o presente estudo a adotar uma análise com painel desbalanceado.

A estratégia geral utilizada para selecionar a melhor especificação do modelo de dados em painel a ser utilizado, com intuito de avaliar os determinantes do índice de dominação nas cooperativas de crédito mineiras filiadas ao Sicoob-Crediminas, consistiu em estimar primeiro os modelos pooled e os efeitos fixos, e então em verificar se o modelo pooled é preferível ao modelo com efeitos fixos utilizando o teste de Chow. No segundo passo, estima-se o modelo com efeitos aleatórios, utilizando-se então o teste de Breusch-Pagan de modo a verificar se o modelo pooled é preferível ao modelo com efeitos aleatórios. O terceiro passo consiste em testar se o modelo com efeitos aleatórios é preferível ao modelo com efeitos fixos, utilizando-se o teste de Hausman. Quando se conclui, com os passos anteriores, que o modelo de efeitos fixos é o melhor, executa-se o quarto passo que consiste em testar se há autocorrelação via aplicação do teste proposto por Wooldrigde (2002), além de se verificar a presença de heterocedasticidade de grupo via utilização do teste de Wald. Finalmente, se a autocorrelação e a heterocedasticidade forem confirmadas, o modelo com efeitos fixos é re-estimado utilizando estimadores Mínimos Quadrados Generalizados Factíveis (Feasible Generalized Least Squares ou FGLS), como sugerido por Judge et al. (1985) e Davidson \& Mackinnon (1993). Observe que Baltagi \& Wu (1999) e Hansen (2007) também utilizaram estimadores FGLS quando detectaram que os erros dos seus modelos de dados em painel não balanceados apresentavam autocorrelação.

A estrutura básica do modelo de regressão em painel utilizada nesta pesquisa para o Sicoob-Crediminas, é dada por:

$$
\begin{aligned}
I D_{i t}=\alpha & +\beta_{T A M} T_{A A}+\beta_{E C S} E_{i t} S_{i t}+\beta_{I D A D E} I D A D E_{i t} \\
& +\beta_{D T I P O} D T I P O_{i t}+\beta_{P L A T} P_{L A T_{i t}} \\
& +\beta_{D E S P A T} D E S P A T_{i t}+\beta_{I N A D} I N A D_{i t} \\
& +\beta_{R E S C A P} R E S C A P_{i t}+v_{i t}+\varepsilon_{i t}
\end{aligned}
$$

Em que: $\mathrm{i}=1, \ldots, 117$ cooperativas filiadas ao Sicoob-Crediminas; $\mathrm{t}=1$, ...,161 meses, referentes ao período de janeiro de 1995 a maio de 2008 das cooperativas filiadas ao Sicoob-Crediminas; ID = Índice de dominação, e constitui a variável dependente do modelo; $\alpha=$ é o intercepto do modelo; $\beta=$ representa os coeficientes angulares estimados para cada variável dependente. As variáveis independentes do modelo são: $\mathrm{TAM}=$ Tamanho $($ proxy $=$ total do Ativo); ECS = Empréstimos sobre o Capital Social; IDADE = Tempo de existência da cooperativa, em anos; DTIPO = assumirá valor 0 se for cooperativa de livre admissão (aberta), ou valor 1 se não for livre admissão (fechada); PLAT = Patrimônio Líquido Ajustado sobre o Ativo Total; DESPAT = Despesas Ope- 
racionais sobre o Ativo Total Médio; INAD = Taxa de inadimplência; RESCAP $=$ Reserva de Capital; e $v_{i}+\varepsilon_{i t}=$ são os resíduos do modelo.

\subsection{Fonte dos Dados.}

O estudo abrange 117 cooperativas localizadas no Estado de Minas Gerais, e estas representam aproximadamente $50 \%$ das cooperativas de crédito mineiras filiadas ao Sicoob-Brasil. Em maio de 2008, consta o funcionamento de 97 cooperativas. Os dados contábeis mensais foram fornecidos pela cooperativa Central Sicoob-Crediminas, os quais foram extraídos do sistema de informações gerenciais e contábeis da mesma e totalizam 16.010 observações, referentes ao período de janeiro de 1995 a maio de 2008. Como os dados são considerados de natureza confidencial, a sua obtenção só é possível mediante a solicitação junto a esta instituição.

\section{Análise e Discussão dos Dados.}

\subsection{Análise de dominação nas cooperativas de crédito mineiras filiadas ao Sicoob-Crediminas}

Nesta seção, serão analisados os resultados obtidos para o Sicoob-Crediminas em relação aos:

1. benefícios monetários líquidos recebidos pelos aplicadores (NMBS)

2. benefícios monetários líquidos recebidos pelos tomadores (NMBB)

3. resultados do "dif" que é a diferença entre o NMBS e o NMBB

4. resultados obtidos através do índice de dominação (ID) para as cooperativas de crédito mineiras

Os benefícios monetários líquidos recebidos pelos aplicadores (NMBS) são medidos pela relação entre as taxas pagas pelas cooperativas de crédito sobre todos os instrumentos de aplicação oferecidos e as melhores alternativas pagas pelo mercado para tipos similares de instrumentos de aplicação. Assim, cooperativas com os NMBS positivos indicam que as mesmas estão oferecendo taxas mais vantajosas aos seus associados, se comparadas às taxas praticadas pelo mercado, e vice-versa. Constatou-se que as cooperativas de crédito singulares filiadas ao Sicoob-Crediminas, no período analisado de janeiro de 1995 a maio de 2008, possuem, em mais de 95,2\% das observações, o NMBS com sinal positivo. Este resultado indica que as cooperativas de crédito singulares filiadas ao Sicoob-Crediminas oferecem maiores vantagens em termos de taxas de aplicações aos seus associados, se comparadas ao mercado bancário tradicional.

O benefício monetário líquido recebido pelos tomadores (NMBB) mede o custo de oportunidade para estes membros da decisão de obter recursos da cooperativa, em vez de obtê-los na melhor fonte alternativa no mercado. O NMBB relaciona então as taxas de mercado para instrumentos de dívida em relação às taxas praticadas pelas cooperativas de crédito, taxas estas ajustadas pela proporção da renda que é restituída aos tomadores da cooperativa em função do fato de os cooperados serem donos das cooperativas e possuírem direitos quando da distribuição de sobras. 
Desta feita, o NMBB positivo indica que as taxas de empréstimos cobradas por outras instituições financeiras são superiores às taxas de empréstimos praticadas pelas cooperativas de crédito, ajustadas pela restituição de juros aos membros tomadores. Se o NMBB é negativo, isto significa que as cooperativas estão praticando taxas sobre instrumentos de dívida menos atrativas, se comparada àquelas praticadas pelo mercado. Os resultados obtidos nesta pesquisa indicam que 90,7\% das observações são de NMBB positivos, sinalizando que os cooperados tomadores possuem na cooperativa de crédito a melhor alternativa para captar recursos.

Apesar de ter sido verificado no presente estudo que as cooperativas filiadas ao Sicoob-Crediminas apresentam vantagens para seus cooperados aplicadores e para os tomadores de recursos em relação ao mercado, Taylor (1971) destaca que existe um conflito potencial entre estes dois grupos de membros, a saber: os tomadores procuram baixas taxas de juros, ao passo que os aplicadores buscam taxas satisfatórias de remuneração sobre suas aplicações. Assim, a análise da diferença entre o NMBS e o NMBB permite verificar se existe algum tipo de dominação nas cooperativas de crédito, seja esta por aplicadores ou por tomadores de recursos. A constatação desta diferença, representada pela abreviação "dif", será posteriormente reavaliada através do índice de dominação (ID) sugerido por Patin \& McNiel (1991a).

Se esta diferença (dif) for positiva, isso indica que a cooperativa de crédito aloca mais benefícios monetários para seus membros aplicadores, mas se a "dif" for negativa, a cooperativa aloca mais benefícios para os membros tomadores. Verificou-se no presente estudo que $88,6 \%$ das observações são de dif $<0$ nas cooperativas de crédito mineiras filiadas ao Sicoob-Crediminas, ou seja, as cooperativas filiadas a este sistema alocam mais benefícios para os membros tomadores de recursos.

Foi verificado ainda se a dif era constituída de amostras normalmente distribuídas, de modo a permitir a implementação do teste " $t$-Student" - para a diferença de médias. Como tanto os testes de normalidade de Jarque \& Bera (1980) - quanto de Doornik \& Hansen (1994) - indicaram que a "dif" não seguia uma distribuição normal, foi aplicado o teste de Wilcoxon para avaliar se havia diferença de medianas, e o teste de Mann-Whitney para avaliar se estas diferenças eram provenientes de uma mesma distribuição de probabilidade. Os resultados destes testes demonstraram que as cooperativas com dif $>0$ e com $\operatorname{dif}<0$ possuem diferenças de medianas estatisticamente significativas e não são provenientes de uma mesma distribuição de probabilidade, o que permite afirmar que existem diferenças entre estes grupos de cooperativas e, ainda, reafirmar que existe dominação de membros tomadores nas cooperativas de crédito mineiras filiadas ao Sicoob-Crediminas.

Neste mesmo contexto, foi seguida a sugestão de Patin \& McNiel (1991a) de criar um índice de dominação (ID), que ajusta para a possibilidade de viés de tamanho e a medida do grau que a cooperativa aloca os benefícios para um grupo de membros específico. O sinal do ID indicará se a cooperativa tende a ser dominada por tomadores (ID $<0)$ ou por aplicadores (ID $>0$ ).

Em $87,3 \%$ das observações, o índice de dominação nas cooperativas filiadas ao Sicoob-Crediminas foi menor que zero $(I D<0)$, o que reforça as análises anteriores, de que existe a dominação de membros tomadores no período estudado. O valor absoluto do índice ID é uma medida relativa do desvio do comportamento de neutralidade nas cooperativas de crédito, ou seja, quanto mais próximo de zero for o ID, mais próximo do comportamento de neutra- 
lidade. Os resultados da proxy ID demonstraram que no Sicoob-Crediminas, esta variação foi de $-9,75$ a 9,99; todavia, os dados concentram-se em valores próximos de zero, ou seja, tem-se a dominação de membros tomadores, mas esta não dista em muito do comportamento de neutralidade.

Assim, assume-se que apesar de ocorrer um comportamento próximo da neutralidade por parte das cooperativas filiadas, tem-se a tendência da dominação de membros tomadores, visto que $87,3 \%$ das observações são valores negativos.

Desta forma, a aceitação da dominação por membros tomadores pode ser justificada por alguns aspectos já previamente discutidos na revisão de literatura, tais como o argumento de Walker \& Chandler (1977) de que, para manter baixas taxas sobre os empréstimos, a cooperativa teria que limitar a capacidade de distribuir as sobras, mas, por outro lado, se a mesma mantivesse altas sobras, seria necessário praticar altas taxas sobre os empréstimos. Assim, de acordo com esses autores, esses dois aspectos - teto máximo para taxa sobre empréstimos e teto máximo para distribuição de sobras - impedem a cooperativa de tratar a competição grupal de modo imparcial na alocação dos benefícios monetários líquidos para os membros, resultando em um viés pró-tomadores.

No Sicoob-Crediminas, que inicialmente era constituído apenas por cooperativas de crédito rural, a dominação de membros tomadores se justifica também pela redução do crédito rural oficial e as mudanças daí decorrentes na década de 90, que tornaram as cooperativas de crédito rural uma alternativa para suprir as demandas de financiamento dos produtores, propiciando, assim, uma orientação para os membros tomadores de recursos.

\subsection{Determinantes do índice de dominação nas cooperativas de crédito mineiras filiadas ao Sistema Sicoob-Crediminas}

Após a constatação de que as cooperativas de crédito mineiras filiadas ao Sicoob-Crediminas são dominadas por membros tomadores, serão avaliados quais são os determinantes da dominação nestas cooperativas filiadas.

A variável independente "Reserva de capital - RESCAP" descrita na equação 7 foi excluída do modelo pelo fato de apresentar alta correlação com a variável "Patrimônio Líquido Ajustado sobre o Ativo Total - PLAT".

Na Tabela 2 são apresentadas as variáveis empregadas no modelo de dados em painel para avaliar os determinantes do índice de dominação, juntamente com suas estatísticas descritivas.

Os resultados obtidos no modelo de dados em painel, estimados para determinar o índice de dominação das cooperativas filiadas ao Sicoob-Crediminas, estão descritos na Tabela 3. De início, nota-se que a variável "tamanho - TAM" apresentou um coeficiente de valor zero, o que reflete uma influência não representativa sobre o índice de dominação das cooperativas filiadas ao SicoobCrediminas (Tabela 3).

A variável "Empréstimo/Capital Social - ECS" foi estatisticamente significativa a $1 \%$ para explicar o índice de dominação, indicando que o aumento do indicador ECS reduz o índice de dominação nas cooperativas de crédito. $\mathrm{O}$ aumento de $10 \%$ no índice ECS gera uma redução de 0,00685 no ID das cooperativas filiadas ao Sicoob-Crediminas, ou seja, o impacto é relativamente pequeno, e a magnitude de variação do ID é de -9,75 a 9,99 nas cooperativas filiadas ao Sicoob-Crediminas. 
Tabela 2: Estatística descritiva das variáveis empregadas no modelo para avaliar os determinantes do índice de dominação nas 117 cooperativas de crédito mineiras filiadas ao Sicoob-Crediminas, no período de janeiro de 1995 a maio de 2008.

\begin{tabular}{lrrrr}
\hline Variável & \multicolumn{1}{c}{ Média } & \multicolumn{1}{c}{ Desvio-Padrão } & \multicolumn{1}{c}{ Mínimo } & \multicolumn{1}{c}{ Máximo } \\
\hline ID & $-0,32$ & 0,94 & $-9,74$ & 9,99 \\
TAM & $8.235 .865,00$ & $11.600 .000,00$ & 625,00 & $167.000 .000,00$ \\
ESC & 6,29 & 18,26 & 0 & $1.348,46$ \\
Idade & 10,58 & 5,36 & 0,16 & 38,02 \\
DTIPO & 0,89 & 0,31 & 0 & 1 \\
PLAT & 0,24 & 0,28 & $-8,14$ & 3,05 \\
DESPAT & 0,03 & 0,04 & 0 & 1,99 \\
INAD & 0,05 & 0,07 & 0 & 1,05 \\
\hline
\end{tabular}

Fonte: Resultados da pesquisa

Tabela 3: Determinantes do índice de dominação nas cooperativas filiadas ao SicoobCrediminas, no período de janeiro de 1995 a maio de 2008.

\begin{tabular}{|c|c|}
\hline Variáveis & Coeficiente \\
\hline TAM & $\begin{array}{l}0,0000^{* * *} \\
(0,0000)\end{array}$ \\
\hline ECS & $\begin{array}{c}-0,0685^{* *} \\
(0,0041)\end{array}$ \\
\hline IDADE & $\begin{array}{c}-0,0238^{* * *} \\
(0,0044)\end{array}$ \\
\hline DTIPO & $\begin{array}{l}0,0408^{* *} \\
(0,0201)\end{array}$ \\
\hline PLAT & $\begin{array}{c}-1,9914^{* * *} \\
(0,1179)\end{array}$ \\
\hline DESPAT & $\begin{array}{l}0,3989^{\text {ns }} \\
(0,2947)\end{array}$ \\
\hline INAD & $\begin{array}{l}0,2460^{* *} \\
(0,0972)\end{array}$ \\
\hline Constante & $\begin{array}{l}0,8941^{* *} \\
(0,0978)\end{array}$ \\
\hline \multicolumn{2}{|c|}{$\mathrm{N}^{\mathrm{o}}$ Obs. $=9.642$} \\
\hline \multicolumn{2}{|c|}{$\mathrm{N}^{\mathrm{o}}$ de grupos $=112$} \\
\hline \multicolumn{2}{|c|}{$\mathrm{N}^{\mathrm{o}}$ mínimo de observações por grupo: 2} \\
\hline \multicolumn{2}{|c|}{ Wald $\chi^{2}(118)=1.910,75$ Prob $>\chi^{2}=0,000$} \\
\hline \multicolumn{2}{|c|}{$\begin{array}{l}\text { Obs.: Estimação por FGLS, assumindo dummies para } \\
\text { os efeitos fixos e assumindo os problemas de } \\
\text { heterocedasticidade e autocorrelação detectados } \\
\text { através dos procedimentos operacionais. } \\
* * *, * * \mathrm{e}^{*} \text { implicam significância estatística aos níveis } \\
1 \%, 5 \% \text { e } 10 \% \text {, respectivamente ( ) indica o } \\
\text { erro-padrão } \\
\text { Fonte:Resultados da pesquisa }\end{array}$} \\
\hline
\end{tabular}


O aumento da variável ECS pode ser devido ao aumento do volume de empréstimos ou a uma redução do capital social relativa ao volume de empréstimos. Estas características são condizentes com uma maior alocação de benefícios monetários líquidos para o tomador, que é representada pela redução do índice de dominação.

A variável "IDADE" foi estatisticamente significativa a 1\% para explicar o índice de dominação nas cooperativas filiadas ao Sicoob-Crediminas (Tabela 3). O aumento de um ano nas cooperativas de crédito filiadas reduz em 0,0238 o índice de dominação, ou seja, quanto mais antiga a cooperativa, menor o índice de dominação, e, consequentemente, tem-se a dominação por tomadores. Como no Sicoob-Crediminas, as cooperativas são dominadas por tomadores, este resultado indica que o índice de dominação é maior nas cooperativas com menor tempo de existência, mas ainda com o ID $<0$.

A variável "DTIPO" foi significativa a 5\% para explicar o ID nas cooperativas filiadas ao Sicoob-Crediminas, e apresentou relação positiva com o índice de dominação (Tabela 3). Ou seja, se a cooperativa não é de livre admissão (cooperativa fechada) o índice de dominação tende a aumentar em 0,0408. Este resultado diverge daquele do estudo prévio de Leggett \& Stewart (1999), que afirma que as cooperativas de livre admissão (cooperativas abertas) tendem a ser dominadas por tomadores, ao passo que aquelas que não são de livre admissão (cooperativas fechadas) tendem a ser dominadas por aplicadores. Para o presente estudo, cabe destacar que as cooperativas filiadas ao Sicoob-Crediminas são formadas por membros tomadores em sua essência, o que impossibilita a captação desta diferenciação na estimação do modelo econométrico.

Outra questão importante no caso do Sicoob-Crediminas, e que ajuda a entender a não captação de diferenciação pelo modelo econométrico estimado, é que as análises se iniciaram em janeiro de 1995 e, apesar de em maio de 2008 haver uma proporção de $53 \%$ das cooperativas analisadas classificadas como de livre admissão, estas passaram a esta condição somente depois de 2004, o que implica um período muito maior de observações com estas cooperativas classificadas como fechadas, como se pode notar pela Tabela 4.

Tabela 4: Classificação de livre admissão ou não e número de cooperativas filiadas ao Sicoob-Crediminas, no período de janeiro de 1995 a maio de 2008.

\begin{tabular}{lcc}
\hline Classificação das cooperativas & Número de cooperativas & Percentual \\
\hline Livre Admissão em 2004 & 10 & $8,5 \%$ \\
Livre Admissão em 2005 & 24 & $20,5 \%$ \\
Livre Admissão em 2006 & 11 & $9,4 \%$ \\
Livre Admissão em 2007 & 14 & $12,0 \%$ \\
Livre Admissão em 2008 & 3 & $2,6 \%$ \\
Cooperativas Fechadas & 55 & $47,0 \%$ \\
\hline Total de cooperativas & 117 & $100,00 \%$ \\
\hline Fonte: Resultados da pesquisa & &
\end{tabular}

A variável "Patrimônio Líquido Ajustado/Ativo Total - PLAT" foi estatisticamente significativa - a 1\% para explicar o índice de dominação no SicoobCrediminas (Tabela 3). Esta variável apresentou sinal negativo, indicando que o aumento de 0,1 do indicador PLAT reduz o índice de dominação nas cooperativas de crédito em 0,19914 . 
O PLAT informa qual o percentual dos ativos é financiado por recursos próprios da cooperativa (patrimônio líquido), e pode ser considerado o inverso do indicador de alavancagem. Quanto mais alavancada a cooperativa, maior a participação de capitais de terceiros. Por outro lado, quanto menos alavancada a cooperativa, menor é a propensão a captar recursos no mercado e assumir riscos e, portanto, menor a possibilidade de auferir rendimentos. Assim, quanto maior o indicador PLAT, melhor para a cooperativa de crédito, considerando a perspectiva de solvência.

O indicador "Despesas Operacionais/Ativo Total Médio - DESPAT" mede o custo associado com o gerenciamento de todos os ativos da cooperativa de crédito e reflete o grau de eficiência ou ineficiência operacional e, de acordo com a WOCCU, este índice deve ser inferior a 10\% (Richardson 2002). Todavia, o mesmo não se mostrou significativo para explicar o índice de dominação no grupo de cooperativas filiadas ao Sicoob-Crediminas (Tabela 3).

A variável "Inadimplência - INAD" foi significativa a 5\%, e apresentou relação positiva com o índice de dominação (Tabela 3). Ou seja, se a cooperativa aumentar em 0,1 o índice de inadimplência, o índice de dominação tende a se elevar na média em 0,0246 .

A este respeito, a Tabela 5 mostra que, nas cooperativas filiadas ao SicoobCrediminas, a maior média de inadimplência está entre as cooperativas classificadas como dominadas por membros aplicadores. Assim, a variável inadimplência é relevante para a determinação do índice de dominação. Todavia, ressalta-se que em sua essência as cooperativas filiadas a esta central são dominadas por membros tomadores, o que não necessariamente permite afirmar que, neste caso, as cooperativas dominadas por membros tomadores possuem menores percentuais de inadimplência.

Tabela 5: Estatística descritiva para a variável "INAD" por tipo de dominação nas cooperativas filiadas ao Sicoob-Crediminas.

\begin{tabular}{lrccrrr}
\hline Sicoob-Crediminas & Obs. & Média & Desvio-Padrão & Mínimo & Máximo & CV \\
\hline Coop_Tomador & 8.696 & 0,04 & 0,05 & 0 & 1,05 & 1,25 \\
Coop_Aplicador & 978 & 0,12 & 0,16 & 0,004 & 1 & 1,33 \\
Nd. & 6.336 & & & & & \\
\hline Total & 16.010 & & & \\
\hline Resultado do teste t $\Rightarrow$ Ho: Média Coop_aplicador = Média Coop_tomador; \\
Ha : Média Coop_aplicador $>$ Média Coop_tomador. \\
t=32,9934; Valor p = 0,000 \\
\hline CV: coeficiente de variação = desvio-padrão/média \\
Coop_tomador = Cooperativas com ID < 0 \\
Coop_Aplicador = Cooperativas com ID >0 \\
Nd: dados não disponíveis. \\
Fonte:Resultados da pesquisa
\end{tabular}

\section{CONCLUSÕES}

O presente estudo permite inferir que as cooperativas de crédito filiadas ao Sicoob-Crediminas, apresentaram dominação por membros tomadores no período entre janeiro de 1995 e maio de 2008. Este resultado indica que os cooperados utilizam-nas mais para a obtenção do que para a aplicação de recursos financeiros. 
Conforme mencionado, dentro das cooperativas de crédito pode haver grupos com diferentes relações econômicas, as quais podem implicar em certo grau de conflito de interesses entre os membros. Além disso, uma das características das cooperativas não é o lucro individual, mas sim alcançar objetivos econômicos e sociais do grupo. Por esse motivo, o objetivo da cooperativa seria o de engajar em atividades econômicas de maneira a oferecer mais vantagens aos seus cooperados.

Neste contexto, apesar de refutada a hipótese de neutralidade nas cooperativas do sistema Sicoob-Crediminas, não se pode negar que as mesmas estão oferecendo vantagens aos seus cooperados, ao atuarem com taxas sobre empréstimos inferiores às praticadas no sistema bancário, e também maiores taxas de remuneração sobre os recursos aplicados na cooperativa, fatos que foram evidenciados pelas análises dos benefícios monetários líquidos para os membros tomadores (NMBB) e também nas análises dos benefícios monetários líquidos para os membros aplicadores (NMBS). Complementarmente, os resultados do Índice de Dominação (ID) indicam a existência de um comportamento de dominação não muito intensificado e próximo da neutralidade, o que pode estar associado ao fato de haver grande concentração de observações deste índice em valores próximos de zero.

Como determinantes para este comportamento tomador, foram verificadas relações significativas para a redução do ID nas variáveis Empréstimo/Capital Social (ECS), Idade e Patrimônio Líquido Ajustado/Ativo Total (PLAT). Já as variáveis Inadimplência (INAD) e DTIPO (variável dummy que caracteriza a cooperativa como sendo de livre admissão - DTIPO $=1-$ ou não - DTIPO =0) apresentaram relação positiva com o Índice de Dominação no período analisado. Todavia, os resultados obtidos para este modelo - em especial para as duas variáveis com relação positiva - devem ser confirmados por novos estudos, que analisem a relação entre a dominação e o crescimento recente das cooperativas de livre admissão observado a partir de 2003 no conjunto das cooperativas filiadas ao sistema Sicoob-Crediminas, e a influência dos índices de inadimplência dentro do conjunto das cooperativas dominadas por membros tomadores.

Recomenda-se também, para trabalhos futuros, avaliar se as alterações no número de membros afetam o tipo de dominação nas cooperativas de crédito, verificar se existem diferenças significativas entre os índices de dominação nos sistemas Sicoob, Sicredi, Unicred, Ancosol, Confesol, e Cooperativas Independentes existentes no Brasil, identificar as implicações desta dominação de membros tomadores nas formas de gerenciamento e obtenção de ganhos por parte das cooperativas de crédito. Neste sentido, pode-se analisar até que ponto este comportamento está comprometendo a sustentabilidade financeira destas organizações, que desempenham um papel importante no sistema de microcrédito nacional. Ainda, as análises desenvolvidas neste trabalho podem ser confrontadas no intuito de verificar se existem diferenças significativas entre os estados da federação e os diferentes sistemas de crédito cooperativo. Adicionalmente, é possível confrontar e avaliar os resultados da dominação nas cooperativas de crédito assumindo a hipótese - que não foi testada no presente estudo - de que os benefícios monetários e não monetários para os associados das cooperativas de crédito são independentes. 


\section{Referências Bibliográficas}

Baltagi, B. H. \& Wu, P. X. (1999), 'Unequally spaced panel data regressions with $\operatorname{ar}(1)$ disturbances', Econometric Theory 15(6), 814-823,.

BANCO CENTRAL DO BRASIL. Resolução 3.106/2003. (2007), http://www.bcb.gov.br/pre/denor/port/2003/1/.asp.

Black, H. \& Dugger, R. H. (1981), 'Credit unions: growth, competition and regulatory problems', Journal of Finance 36(2), 529-538.

Bonus, H. \& Schmidt, S. (1990), 'The cooperative banking group in the federal republic of germany', Journal of Institutional and Theoretical Economics 146, 180-207.

Davidson, R. \& Mackinnon, J. G. (1993), Estimation and Inference in Econometrics, New York: Oxford University Press.

Doornik, J. A. \& Hansen, H. (1994), An omnibus test for univariate and multivariate normality. Working Paper, Nuffield College, Oxford.

URL: www.doornik.com/research/normal2.pdf

Emmons, W. R. \& Mueller, W. (1997), Conflict of interest between borrowers and lenders in credit cooperatives: The case of german cooperative banks. Working Paper 98-009A, Federal Reserve Bank of St. Louis.

URL: http://research.stlouisfed.org/wp/1997/97-009.pdf

Fischer, K. P. (2000), Deposit insurance and moral hazard in financial cooperatives. Center de recherche en économie et finance appliquées (CREFA), Université Laval, Quebec, Canada.

Fischer, K. P. \& Desrochers, M. (1999), Cooperativas Financieras Y Desarrollo Rural, chapter Costos de agencia y la crisis de cooperativas financieras en Colombia, pp. 45-90. Instituto de Estudios Rurales (IER), Universidad Javeriana, Bogotá.

Goddard, J. \& Wilson, J. O. S. (2005), 'Us credit unions: An empirical investigation of size, age and growth.', Annals of Public and Cooperative Economics 76(3), 375-406.

Gujarati, D. (2006), Econometria Básica, 4 edn, Campus.

Hansen, C. B. (2007), 'Generalized least squares inference in panel and multilevel models with serial correlation and fixed effects', Journal of Econometrics 140, 670-694.

Jarque, C. M. \& Bera, A. K. (1980), 'Efficient tests for normality, homoscedasticity and serial independence of regression residuals', Economics Letters 6(3), 255-259.

Judge, G. G., Griffiths, W. E., Hill, R. C. \& Lütkepohl, H. (1985), The Theory and practice of Econometrics, 2 edn, New York: Wiley.

Kohers, T. \& Mullis, D. (1990), 'The impact of a financial institution's age on its financial profile and operating characteristics: the evidence for the credit union industry', Review of Business and Economic Research 26, 38-49. 
Leggett, K. J. \& Stewart, Y. H. (1999), 'Multiple common bond credit unions and the allocation of benefits.', Journal of Economics and Finance 23(3), 235245.

Patin, J. R. P. \& McNiel, D. W. (1991a), 'Benefit imbalances among credit union member groups: evidence of borrower-dominate, saver-dominated and neutral behaviour?', Applied Economics Letters 23(4B), 769-780.

Patin, J. R. P. \& McNiel, D. W. (1991b), 'Member group orientation of credit unions and total member benefits', Review of Social Economy 49(1), 37-61.

Richardson, D. C. (2002), 'Pearls monitoring system'.

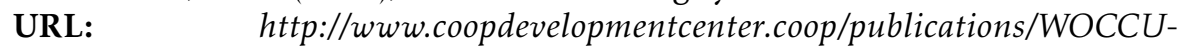
\%20Files/pearlsvol4.pdf

Smith, D. J. (1984), Journal of Finance, Vol. 39, The American Finance Association, chapter A theoretic framework for the analysis of Credit Union decision Making, pp. 1155-1168.

Smith, D. J. (1986), 'A test for variant objective functions in credit unions', Applied Economics 18(9), 959-970.

Smith, D. J., Cargill, T. F. \& Meyer, R. A. (1981), Journal of Finance, Vol. 36, 2 edn, The American Finance Association, chapter An Economic Theory of a Credit Union., pp. 519-528.

Soares, M. M. \& Melo Sobrinho, A. D. (2007), 'Microfinanças: o papel do Banco Central do Brasil e a importância do cooperativismo de crédito', Brasília: BCB.

Spencer, J. E. (1996), Review of Social Economy, Vol. 54, Routledge, chapter An extension to Taylor's Model of Credit Unions., pp. 89-98.

Taylor, R. A. (1971), Review of Social Economy, Vol. 29, Routledge, chapter The Credit Union as a cooperative institution, pp. 207-217.

Taylor, R. A. (1979), 'Demand for labour by credit unions.', Applied Economics 11(3), 333-340.

Walker, M. C. \& Chandler, G. G. (1977), Review of Social Economy, Vol. 35, Routledge, chapter On the allocation of the net monetary benefits of Credit Union membership., pp. 159-168.

Wooldrigde, J. M. (2002), Econometric Analysis of Cross Section and Panel Data., Cambrigde, MA: MIT Press,. 